\title{
CONSIDERATIONS OF VISUAL QUALITY IN OCULAR SURGERY AS PRESENTED AT DRY EYE UNIVERSITY
}

\author{
Frank W. Bowden, III, MD, FACS \\ Board-certified ophthalmologist and founder and medical director of Bowden Eye \& Associates in \\ Jacksonville, FL.
}

Visual quality may be described as the visual experience which reflects the optimal optical efficiency of the eye. Patients undergoing ocular surgery expect improvement in visual acuity. Ocular surgeons typically aim to improve best-corrected visual acuity. Refractive surgeons, on the other hand, strive to improve uncorrected visual acuity. It is not uncommon that patients with excellent corrected visual acuity following surgery may be dissatisfied with visual quality which is less than anticipated due to unexpected visual disturbances. These visual phenomena may include ghosting, glare, halos, reduced contrast sensitivity, and visual fluctuation.

Structural determinants of visual quality may include ocular surface disease, media opacities, disorders of the macula, and optic neuropathy. In the case of macular disorders and optic nerve disease, contrast sensitivity loss accounts for qualitative visual impairment. Careful preoperative ocular examination with diagnostic imaging may effectively identify these disorders to properly set patient expectations following surgery. Patients must be advised that comorbid posterior segment pathology may impact both visual acuity and visual quality following surgery. Commonly encountered posterior segment disorders include epimacular membrane, cystoid macular edema, age-related macular degeneration, macular ischemia, glaucoma, and optic neuropathy.

Media opacities may significantly impact visual quality by causing scatter and degradation of incident light energy transmission to the retina. Corneal opacities and lens opacities are clinically evident before proposed ocular surgery. However, in the case of cataract surgery with lens implant, the optical properties of the lens and lens centration may define optical quality. Intraocular lens design features that affect optical quality include spherical aberration, diffractive optics, and extended depth of focus. When cataract surgery results in the desired visual acuity outcome, it must be realized that residual corneal opacities and aberrations may reduce visual quality. Presbyopia correcting intraocular lens implant design features such as apodization with diffractive optics as well as extended depth of focus (EDOF) may achieve a greater range of visual correction, albeit, with a variable sacrifice in visual quality. Intraocular decentration may also induce higher-order aberrations, primarily coma. ${ }^{1}$ These alterations reduce effective transmission of light energy to the fovea creating higher-order aberrations wherein the clinical effect may be exacerbated by residual ametropia and by mesopic pupil enlargement. Persistent awareness of these visual disturbances may range from a mild nuisance to functional disability.

Presbyopia correction with these lenses may be associated with increased distance photic phenomena as near visual performance improves. ${ }^{2}$ If the patient is unable to neuro-adapt and tolerate the unanticipated photic disturbances, which degrade visual quality, then intraocular lens removal and exchange may be necessary. Preoperative screening with tools such as the Dell questionnaire may help identify patients who may be unreceptive to undesirable visual disturbances following surgery. 
Spherical aberration values of the intraocular lens may be positive, negative, or zero by design. Aspheric lenses have a spherical aberration (SA) value which ranges from zero to -0.27 microns. ${ }^{3}$ Spherical lenses have an SA value greater than zero. The average corneal SA through a $6 \mathrm{~mm}$ pupil is +0.27 microns. The SA of the cornea and the intraocular lens will have an additive effect on the visual system of the eye creating total ocular SA. Optimal visual quality with high optical resolution is realized when the total ocular SA is zero. ${ }^{4}$ However, this is necessarily accompanied by a depth of focus loss. On the other hand, residual SA of the eye which may improve depth of focus may create intolerable glare and halos, particularly under mesopic situations with pupil enlargement. Intraocular lens decentration may induce higher-order aberrations, predominantly coma. This may be avoided by selecting zero aspheric lenses wherein there is no change in power from centre to edge of the lens optic.

The tear film and ocular surface represent the most important determinant of visual quality. A stable tear film and a smooth regular ocular surface are requisite for optimal visual resolution. Corneal surface irregularities and opacities may create visual alterations based on scatter of light energy within the eye. Degraded image quality may be represented by static scatter using the HD Analyzer (Visometrics), which measures the objective scatter index in a time-averaged fashion. The inter blink variation in the higher-order aberrations arising from the unstable tear film defines visual fluctuation, a hallmark feature of dry eye disease. Tear film instability may also be detected by corneal topography which may show distortion of placido disc rings or smeared colour LED images. The unstable tear film may also be captured with the NIKBUT display and graph of the Keratograph 5 (Oculus). Fluorescein staining of the tears may also illustrate tear film dynamics during slit-lamp examination of the ocular surface.

The tear film and ocular surface must be optimized before ocular surgery, as they may be destabilized postoperatively. Topical antiseptics, anesthetics, NSAIDS, and preserved medications used in the perioperative and postoperative periods may disrupt the tear film and injure the ocular surface with degraded visual quality. Corneal incisions, ocular exposure, and surface irrigation further disturb tear film stability. Measures to address ocular surface disease in general and dry eye disease, in particular, are required to realize refractive accuracy in anterior segment surgery and drive patient satisfaction. Basic measures include lubrication, lid hygiene measures, thermal lid care, and immunomodulatory therapy. In those situations where significant ocular surface inflammation is evident, topical steroids and cryopreserved amniotic membrane therapy may be warranted. When significant meibomian gland dysfunction with obstruction, is present, meibomian gland probing, thermal pulsation treatment, and intense pulsed light photo facial therapy with gland expression may be very effective measures to stabilize the tear film and improve the ocular surface.

Visual quality following ocular surgery should not be taken for granted. Recognition of relevant clinical findings and lens implant design features must be considered preoperatively to facilitate meaningful discussion with patients to set realistic expectations for their surgical experience. Ocular surface optimization must be established to ensure optimal visual quality and patient satisfaction.

\section{REFERENCES}

1. Tarib J, Kaiser, I, Herber C, Hagen P, et al. Comparison of visual outcomes and patient satisfaction after bilateral implantation of an EDOF IOL and a mix and match approach. J Refract Surg 2019;35(7):408-16.

2. Ferreira TB, Rebeiro FJ. Prospective comparison of clinical performance and subjective outcomes between two diffractive trifocal intraocular lenses in bilateral cataract surgery. J Refract Surg 2019;35(7):418-25.

3. He JC, Gwiazda J, Thorn F, Held R. Wavefront aberration in the anterior corneal surface and the whole eye. J Opt Soc Am A Opt Image Sci Vis 2003;20:1155-63.

4. Bellucci R, Morselli S, Piers P. Comparison of wavefront aberrations and optical quality of eyes implanted with five different intraocular lenses. J Refract Surg 2004;20:297-306. 